\title{
Plantaciones de coníferas exóticas en Patagonia: los riesgos de plantar sin un manejo adecuado
}

\author{
Estela RafFaele ${ }^{\bowtie}$; Martín A. Núñez \& María A. Relva \\ Laboratorio Ecotono, Universidad Nacional del Comahue, INIBIOMA-CONICET. Bariloche, Argentina.
}

\begin{abstract}
RESUMEN. En la última década se realizaron en la región patagónica una gran cantidad de investigaciones evaluando el impacto ecológico de las plantaciones y analizado los modos de producción con el fin de aumentar la cosecha y reducir su impacto. Si bien las plantaciones brindan beneficios para las economías regionales, a la fecha no está clara la magnitud de esta contribución. Por otro lado, los problemas ambientales que producen las plantaciones son varios; en particular, las invasiones de ecosistemas aledaños es uno de los más importantes en el largo plazo. Otro problema relacionado a las plantaciones, aunque incipiente aún en la región, es el aumento de los incendios forestales. Las plantaciones acumulan gran cantidad de combustible seco e inflamable, especialmente cuando no son manejadas. Además, muchas especies o variedades de pinaceas están adaptadas al fuego, por lo que no sólo podrían cambiar el régimen y el tipo de incendios, sino también retroalimentarse positivamente, ocasionando altos costos sociales, económicos y ecológicos. Consideramos que estos problemas podrían ser minimizados en gran parte con la aplicación de buenas prácticas forestales.
\end{abstract}

[Palabras clave: pinos, invasión, fuego, impacto ambiental]

AвSTRACT. Non-native conifer plantations in Patagonia: risks of planting without proper management: In the last decade in Patagonia, a significant number of research studies evaluated the environmental impact of plantations and analyzed the modes of production in order to increase the yield and reduce its impact. While plantations provide benefits for regional economies, at the present the extent of this contribution is unclear. Furthermore, there are several environmental problems produced by plantations, with the invasions of adjacent ecosystems being one of the most damaging impacts in the long term. Another problem related to the plantations, although still incipient in the region, is the increment of forest wildfires. Plantations accumulate large amounts of dry and flammable fuel, particularly when they are not properly managed. Also many species or varieties of Pinaceae are adapted to fire, so they could not only change the regime and type of fires, but also display positive feedbacks causing high social, economic and ecological costs. We believe that these problems could be minimized to a great extent with the application of good forestry practices.

[Keywords: pines, invasion, fire, environmental impact]

\section{INTRODUCCIÓN}

Las prácticas forestales con coníferas tienen una larga historia en el hemisferio norte. Sin embargo, recién a mediados del siglo pasado se aplicaron a gran escala en Europa, y posteriormente se expandieron a otras partes del mundo. Muchas especies exóticas de árboles han sido plantadas con diversos propósitos, incluyendo la producción de leña y el control de la erosión del suelo en zonas muy degradadas (Richardson 1998). Las plantaciones de especies exóticas contribuyen de forma significativa a las economías de varios países. A pesar de ello, existen altos costos asociados a su uso extensivo, ya estudiados en otros países (Richardson 1998). Una considerable atención tiene, por ejemplo, el efecto de las plantaciones sobre la biodiversidad en hábitats no boscosos, donde producen cambios en las formas de vida dominantes, reducen la biodiversidad estructural, incrementan la biomasa vegetal

estelaraffaele@gmail.com y perturban la dinámica de la comunidad prevaleciente (Armstrong \& van Hensbergen 1996). También existen antecedentes sobre otros efectos que producen las plantaciones, como, por ejemplo, sobre el suelo, la hidrología (Jobbágy et al. 2013) y sobre las invasiones que producen en los ecosistemas aledaños.

Sabemos que, en general, las especies más invasoras son aquellas más ampliamente sembradas durante mucho tiempo. En especial, el género Pinus es problemático, con al menos 16 especies invasoras en varias zonas del hemisferio sur (Richardson \& Higgins 1998). El problema con la forestación de pináceas es que los atributos que las definen como buenas especies forestales también suelen convertirlas en especies con alta probabilidad de invasión (e.g., su tasa de crecimiento elevada). Muchas de estas especies tienen, además, otros atributos que las convierten en buenas invasoras, como la 
producción de semillas numerosas y pequeñas y un período juvenil corto.

En nuestro país, la actividad forestal se promueve sobre la base de la existencia de superficies extensas, con gran aptitud forestal, donde las especies exóticas alcanzan crecimientos que superan en muchos casos a los de sus zonas de origen, con lo cual se pretende diversificar la producción de la región, cubrir la demanda de madera a nivel regional y disminuir la presión de extracción sobre el bosque nativo (Davel 2008). Como consecuencia de las políticas de promoción, considerables superficies de bosques y de otras comunidades nativas han sido sustituidas por especies exóticas.

En la Patagonia, las políticas forestales privadas y estatales se orientaron durante las últimas décadas hacia la implantación de coníferas exóticas. Estas forestaciones han producido beneficios a la región, pero también han generado problemas. Existe un debate creciente entre las organizaciones ambientalistas, los profesionales, los científicos y los pobladores de la región patagónica acerca del proceso de sustitución de las plantaciones de coníferas exóticas y sus efectos sobre la vegetación nativa (i.e., bosques, matorrales y estepas) y la economía regional. Durante la última década se realizaron y se continúan subsidiando un número elevado de proyectos de investigación que evalúan el impacto ecológico de las plantaciones y analizan los modos de producción con el fin de aumentar la cosecha y minimizar su impacto. También se han escrito diferentes artículos sobre este tema -muchos de ellos científicos y/o técnicos, otros de divulgación-, que son leídos por públicos diferentes, con miradas distintas sobre el tema. Con los años, las discrepancias se han ido multiplicando. Uno de los inconvenientes que vemos es que las prácticas forestales no han sido llevadas a cabo de modo apropiado en varios aspectos, con lo que queda abierta la pregunta: "¿qué pasaría si las forestaciones fueran manejadas adecuadamente?" Sobre la base de nuestra experiencia, a continuación describimos los principales problemas ambientales que generan las plantaciones.

\section{FORESTACIÓN E INVASIÓN}

Si bien el área para plantaciones es potencialmente grande y puede impactar notablemente sobre la diversidad y los servicios ecosistémicos, el espacio donde ocurren o pueden ocurrir las invasiones es aun más grande. Las especies de coníferas más utilizadas en las forestaciones en Patagonia son pino murrayana (Pinus contorta Douglas ex Lauden), pino ponderosa ( $P$. ponderosa Douglas ex C. Lawson) y pino oregón (Pseudotsuga menziesii [Mirb.] Franco), de las cuales se conoce su manejo silvícola e industrial. Estas especies poseen antecedentes de invasión en otras regiones del mundo. Por ejemplo, el pino murrayana ha invadido matorrales, praderas, pasturas y bosques abiertos en Nueva Zelanda. El pino ponderosa ha invadido sitios disturbados en Chile, mientras que en Nueva Zelanda invade praderas y arbustales. Por último, el pino oregón ha invadido bosques mediterráneos en España y también está invadiendo los bosques patagónicos de Chile y Argentina (Simberloff et al. 2003; Peña \& Pauchard 2001; Sarasola et al. 2006; Orellana \& Raffaele 2010).

En Patagonia es común observar, fuera de las plantaciones, la regeneración natural produciendo semillas. Esto se ha observado tanto en la estepa como en el bosque de Austrocedrus chilensis (ciprés de la cordillera) y en los matorrales (Caccia \& Ballaré 1998; Orellana \& Raffaele 2010; Sarasola et al. 2006; Simberloff et al. 2002). Esta capacidad de regenerarse naturalmente podría dar comienzo a procesos de invasión en determinados ambientes, dominando o reemplazando la vegetación nativa. De no ser controlado, este proceso de invasión podría generar importantes cambios en la biodiversidad de la biota, en el funcionamiento de los ecosistemas invadidos, en la estructura del paisaje, en los ciclos naturales del agua, los nutrientes y del fuego, disminuyendo las áreas de pastoreo y representando una amenaza seria para el manejo de las áreas protegidas. Por lo tanto, existe un gran riesgo de que, a mediano plazo, sucedan procesos de invasión a gran escala, lo que tendría efectos ambientales perjudiciales para los ecosistemas locales y, por ende, perjudicando la actividad forestal en general.

A todo esto se suma que los pinos fuera de las plantaciones carecen de valor forestal; al crecer en densidades elevadas, su crecimiento se ve afectado y pueden ser una fuente de plagas y enfermedades. A su vez, la probabilidad de fuegos de copa puede aumentar ya que sirven como escalera de biomasa, e intensifican la competencia por recursos como agua y nutrientes. Además, luego de la cosecha de la forestación, la invasión puede provocar dificultades para retomar la actividad forestal, ganadera y/o 
turística. Desde el punto de vista económico y social, en nuestro país la actividad forestal está promovida y subsidiada por el gobierno nacional (Ley 20580 y Ley 26432) y por los gobiernos provinciales; es considerada un aporte al crecimiento económico y al desarrollo nacional y regional. En el NO de la Patagonia, desde la década del setenta, la tendencia del ritmo de forestación es creciente y el potencial de la región es grande. Estudios de aptitud forestal estiman que la superficie apta para ser forestada es de 800000 ha, y los cálculos más ambiciosos estiman dos millones de hectáreas (Schlichter \& Laclau 1998). Por otro lado, la invasión de pinos podría disminuir la calidad del paisaje para el turismo, actividad que representa aproximadamente $50 \%$ del PBI de esta región cuya principal atracción son los ecosistemas nativos.

La invasión de pinos es relativamente predecible, las fuentes de dispersión de semillas se pueden identificar con facilidad (forestaciones o cortinas), las semillas son dispersadas por el viento y se conoce la edad en la cual las especies producen semillas. Esta alta predictibilidad permite realizar monitoreos y controles preventivos, a diferencia de especies vegetales invasoras dispersadas por mamíferos, aves u otros medios. Además, la etapa actual del desarrollo forestal en Patagonia se presenta como una oportunidad única de evitar una invasión a gran escala (Simberloff et al. 2010). Las invasiones consumadas son difíciles de erradicar y costosas de controlar. En nuestra región, los antecedentes permiten afirmar que aún no estamos ante un proceso de invasión a escala regional; el ritmo de avance de los renovales es un proceso predecible y lento comparado con otras especies invasoras de la región, como la retama (Cytisus scoparius) o la rosa mosqueta (Rosa rubiginosa). Asimismo, la especie de pino más plantada es el pino ponderosa (una de las especies menos invasivas [Sarasola et al. 2006]), y un gran porcentaje de las plantaciones no se encuentra aún en la etapa de producir semillas ( $57 \%$ en 2006) (Loguercio \& Deccechis 2006). Por lo tanto, consideramos que nos encontramos en el momento ideal para monitorear y controlar el proceso de invasión con bajos costos y una alta probabilidad de éxito, ya que el mejor abordaje es la prevención y el control temprano. Es importante notar que a medida que se aumenta el área forestada también aumenta el riesgo de invasión (Simberloff et al. 2010; Núñez et al. 2011), por lo que un aumento de la actividad forestal en el futuro también aumentaría la probabilidad de invasión.

\section{FUEGO E INVASIONES: ¿EFECTOS SINÉRGICOS?}

La mayoría de las especies de pinos plantadas en Patagonia Argentina están adaptadas al fuego en sus lugares de origen. Si bien aún no conocemos el grado de inflamabilidad de estas especies en esta región, es esperable que también sean muy combustibles. Las características más comunes de los árboles adaptados al fuego son aquellas que los hacen sobrevivir a este disturbio (e.g., una corteza gruesa en el tronco) y aquellas que estimulan la germinación de semillas o la floración (Keeley \& Bond 1997; Pausas 2012). Además, hay especies que contienen aceites, resinas $u$ otros compuestos químicos inflamables que, en conjunto, aumentan la probabilidad de incendio en las comunidades donde viven. Estas especies son denominadas pirogénicas y contribuyen sustancialmente a que un sistema sea más inflamable (Pausas et al. 2012), además de poseer una clara ventaja en relación al resto de las especies de la comunidad en condiciones post-fuego.

Ya sea por causas naturales o antrópicas, el fuego forma parte de la dinámica de los ecosistemas del NO de la Patagonia. No obstante, la combinación de fuegos repetidos con las plantaciones de pinos es un nuevo tipo de disturbio. La conversión de grandes áreas de estepa, matorral y bosques de $A$. chilensis en plantaciones de coníferas introducidas en el noroeste patagónico es un cambio reciente en el uso de la tierra. Los cambios estructurales que producen las plantaciones sobre las comunidades nativas son importantes no sólo por la disminución de la biodiversidad local u original de estas comunidades sino también porque pueden afectar los procesos de recolonización después de ocurrido un incendio (Núñez \& Raffaele 2007). Si además le sumamos una invasión potencial de pinos, entonces estos cambios podrían ser irreversibles y, a mediano plazo, ocasionarían pérdidas económicas importantes. Un ejemplo actual sobre plantaciones de pinos quemadas, con posterior invasión de pinos que luego se volvieron a quemar e invadir, es la Reserva de Usos Múltiples Lago Epuyen, en la provincia de Chubut. En esta región existen plantaciones adultas de pino radiata, oregón y murrayana. Muchas de ellas fueron afectadas por varios 
incendios de grandes magnitudes (1987, 1999, 2012 y 2015). Por ejemplo, el incendio de 1987 afectó 8100 ha, de las cuales 4000 ha volvieron a incendiarse en 2012. Entre medio de estos incendios, las diferentes especies de pinos invadieron la zona y la convirtieron en tierras improductivas. En la actualidad, esta zona fue seleccionada por el Observatorio Nacional de la Degradación de Tierras, gestionado por el CONICET, como uno de los sitios piloto para estudiar el deterioro ambiental y socioeconómico de ese lugar. Esto ejemplifica la complejidad del efecto del fuego y su sinergia con las plantaciones e invasiones de coníferas exóticas.

\section{¿Conviene seguir fomentando LAS PLANTACIONES FORESTALES EN EL NORTE DE LA PATAGONIA?}

Consideramos que hoy no es posible responder esta pregunta con la información disponible. Sería interesante hacer un análisis sobre los impactos ecológicos y beneficios económicos en relación a otras prácticas productivas, como la ganadería o el turismo. Sería también interesante ver cómo los subsidios estatales afectan estas ecuaciones. Lo que sí sabemos es que la aplicación de mejores prácticas, que incluyan el manejo de las invasiones, que reduzcan el riesgo de fuego y que seleccionen de forma correcta el lugar donde plantar, minimizarían el impacto de las plantaciones. Por ejemplo, en zonas poco propensas a las invasiones debería ser rutina usar especies con poco potencial invasor y realizar un seguimiento de las invasiones. El subsidio a las plantaciones (Ley 20580 y Ley 26432) debería incluir fondos para que se realicen controles de invasión, así como asignar fondos para realizar las podas y raleos necesarios, además de incluir los costos asociados a la fiscalización de dichas tareas.

Por último, se deberían capitalizar las experiencias de otras regiones del mundo. Si bien las plantaciones no han sido prohibidas en ningún país, se han mejorado notablemente las prácticas forestales, siendo más cuidadosas con la prevención del fuego, las invasiones y el medio ambiente.

Agradecimientos: Agradecemos a Esteban Jobbágy, Juan Gowda y Adriana Ruggiero la invitación de participar en esta nueva sección: Foro Ecología y Sociedad de la Revista Ecología Austral, para escribir sobre uno de los temas más actuales que tenemos en el noroeste patagónico que son las forestaciones comerciales de coníferas exóticas.

\section{BibLIOGRAFÍA}

Armstrong, AJ \& HJ Van Hensbergen. 1996. Impacts of afforestation with pines on assemblages of native biota in South Africa. South African Forestry Journal, 17:35-42.

CACCIA, FD \& CL BALLARÉ. 1998. Effects of tree cover, understory vegetation, and litter on regeneration of Douglas-fir (Pseudotsuga menziesii) in southwestern Argentina. Canadian Journal of Forest Research-Revue Canadienne De Recherche Forestiere, 28:683-692.

DAVEL, M. 2008. Estimación de la productividad de sitio. Pp. 30-42 en: Davel, M (ed.). Establecimiento y manejo del pino oregón en Patagonia. Esquel, Argentina: CIEFAP.

Jobbágy, EG; AM Acosta \& MD Nosetto. 2013. Rendimiento hídrico en cuencas primarias bajo pastizales y plantaciones de pino de las sierras de Córdoba (Argentina). Ecol. Austral, 23:87-96.

Keeley, JE \& WJ Bond. 1997. Convergent seed germination in South African fynbos and Californian chaparral. Plant Ecol., 133:153-167.

Loguercio, GA \& F Dececchis. 2006a. Forestaciones en la Patagonia Andina: potencial y desarrollo alcanzado. Parte 1. Patagonia Forestal, 1:4-8.

Núñez, MA \& E Raffaele. 2007. Changes due to afforestation on post fire regeneration in native shrubland communities in Northwestern Patagonia, Argentina. J. Veg. Sci., 18:827-834.

NúÑEZ, MA; A MoretTi \& D Simberloff. 2011. Propagule pressure hypothesis not supported by an 80-year experiment on woody species invasion. Oikos, 120: 1311-1316.

Orellana, I \& E Raffaele. 2010. The spread of the exotic conifer Pseudotsuga menziesii in Austrocedrus chilensis forests and shrublands in northwestern Patagonia, Argentina. New Zealand of Forestry Science, 40:199-209.

PAusas, J. 2012. Incendios Forestales. CSIC y Libros de la Catarata. España.

Pausas, J; G Alessio; B Moreira \& G Corcobado. 2012. Fires enhance flammability in Ulex parviflorus. New Phytol., 193:18-23.

Peña, E \& A Pauchard. 2001. Coníferas introducidas en áreas protegidas: un riesgo para la biodiversidad. Bosque Nativo, 30:3-7.

RICHARDSON, DM \& SI HIGGINs. 1998. Pines as invaders in the southern hemisphere. En: Richardson, DM (ed.). Ecology $\mathcal{E}$ Biogeography of Pinus. Cambridge University Press.

RichARDSON, D. 1998. Forestry trees as invasive aliens. Conserv. Biol., 12:18-26.

SARASOLA, MM; VE Rusch; TM SChlichter \& CM GHERSA. 2006. Invasión de coníferas forestales en áreas de estepa y bosques de ciprés de la cordillera en la Región Andino Patagónica. Ecol. Aust., 16(2):143-156.

SChlichter, T \& P LAClau. 1998. Ecotono estepa-bosque y plantaciones forestales en la Patagonia. Ecol. Aust., 8:285-296.

SimberlofF, D; MA Relva \& MA NúÑ̃z. 2002. Gringos en el bosque: introduced tree invasion in a native Nothofagus/ Austrocedurs forest. Biol. Invasions, 4:35-53.

SimberlofF, D; MA RelVA \& MA NúÑEz. 2003. Introduced Species and Management of a Nothofagus/Austrocedrus Forest. Environ. Manage., 31:263-275.

SimberlofF, D; MA NúÑez; NJ Ledgard; A Pauchard; D RICHARDSON; ET AL. 2010. Spread and impact of introduced conifers in South America: Lessons from other southern hemisphere regions. Austral Ecol., 35:489-504. 